\title{
Higher accuracy output feedback sliding mode control of sampled-data systems
}

\author{
Thang Nguyen, Wu-Chung Su, Zoran Gajic, and Christopher Edwards
}

\begin{abstract}
The problem of output feedback sliding mode control for sampled-data systems in the presence of external disturbances is considered. The proposed output feedback control strategy helps obtain a quasi sliding mode with an $O\left(T^{3}\right)$ boundary layer, where $T$ is the sampling period. This outperforms the $O\left(T^{2}\right)$ result induced by the one-step delayed disturbance approximation method. The proposed scheme is applicable to linear systems which are relative degree one and minimum phase. An example is given to illustrate the efficacy of the new method.
\end{abstract}

Index Terms-Sliding mode control; Sampled-data systems; Output feedback; Singular perturbation.

\section{INTRODUCTION}

In practice, the behaviors of dynamical systems are affected by uncertainties and external disturbances. These undesired factors can be mitigated by sliding mode control. In continuous-time systems, matched external disturbances or uncertainties can be rejected using discontinuous control action with infinite switching frequency. In sampled-data systems, because of the nature of the sample/hold, infinite frequency switching cannot be realized. Therefore, an ideal sliding mode cannot be obtained. Instead, "quasi sliding modes", where the system state is kept only in a boundary layer of the sliding surface, takes place [1].

In [2], a non-switching control method for a class of sampled-data systems was exploited to avoid the chattering phenomena during the quasi sliding mode phase. In the state feedback sliding mode control problem, a dead-beat type control law based on the one-step delayed disturbance approximation method results in a quasi sliding mode boundary layer of thickness $O\left(T^{2}\right)$, where $T$ is the sampling period [2]. With this accuracy of quasi sliding mode, the state is kept in ultimate $O(T)$ bound [3]. An $O\left(T^{2}\right)$ quasi sliding mode can be obtained in sampled-data systems in the context of state feedback [3].

The problem of improving system performances, including the boundary layer around the sliding modes and the bound

This work was supported in part by the Vietnam Education Foundation (VEF) and by the Ministry of Science and Technology of Taiwan under project number: 104-2221-E-005 -044 -. Corresponding author: Wu-Chung Su.

Thang Nguyen is with School of Electrical Engineering, International University-Vietnam National University HCMC (email: thangnt@hcmiu.edu.vn).

Wu-Chung Su is with the Department of Electrical Engineering, National Chung-Hsing University 250 Kuo-Kuang Road, Taichung, Taiwan, Republic of China (email: wcsu@nchu.edu.tw).

Zoran Gajic is with the Department of Electrical Engineering, Rutgers University, 94 Brett Road, Piscataway, NJ 08854-8058, USA (email: gajic@ece.rutgers.edu).

Christopher Edwards is with the Department of Engineering, the University of Exeter, Exeter, the United Kingdom (email: C.Edwards@exeter.ac.uk). on the state vector when implementing a non-switching sliding control law, has been recently addressed in [2], [4]. Although the state feedback problem has been extensively studied, only a few authors have considered the output feedback case [5], [4], [6] for sampled-data systems. A discrete-time integral sliding mode control method was proposed to estimate the state variables and external disturbances using two observers[7]. In contrast, no observer was employed in [4], and instead the equivalent control estimation techniques were proposed in [2] and [3] incorporating the unknown quantities both from the external disturbances and the unmeasurable state variables. An $O\left(T^{2}\right)$ boundary layers in the sliding mode and $O(T)$ bounds on state regulation were obtained using the approaches in [4]. A better quasi-sliding mode with $O\left(T^{3}\right)$ accuracy was achieved for single-input single-output systems using a minimum variance control scheme in [8] but an ultimate bound on the state variables was not studied.

In this paper, higher accuracies in the quasi sliding mode and in the bound on the state vector variables are obtained using a dynamic output feedback strategy, which extends the results in [4]. Here, the effect of unknown disturbances is compensated using system information from two previous time instants. A preliminary result along these lines was reported in [9]. A similar compensation technique for a singleinput single-output velocity servo system has been recently reported in [6]. The method in the paper outperforms other approaches dealing with the same problem [7], [4]; and an $O\left(T^{3}\right)$ boundary layer for the quasi sliding mode and an $O\left(T^{2}\right)$ thickness of the state vector is obtained. (The quantities for the methods in [7], [4] are $O\left(T^{2}\right)$ and $O(T)$ respectively.)

Throughout the paper, $\lambda\{A\}$ denotes the spectrum of matrix $A$, while $I_{m}$ stands for an identity matrix of order $m$. A vector function $f(t, s) \in R^{n}$ is said to be $O(s)$ over an interval $\left[t_{1}, t_{2}\right]$, [10], if there exist positive constants $K$ and $s^{*}$ such that $\|f(t, s)\| \leq K s, \quad \forall s \in\left[0, s^{*}\right], \quad \forall t \in\left[t_{1}, t_{2}\right]$. In addition, $f[k]$ stands for $f(k T)$, where $k=0,1,2, \ldots$ indicating the index of the discrete-time sequence.

The structure of the paper is organized as follows. Section II presents the formulation of the problem, followed by Section III where the main results are described. A numerical example is considered to illustrate the effectiveness of the approach in Section IV. Conclusions are given in the final section.

\section{PROBLEM FORMULATION}

Consider a linear system described by

$$
\begin{aligned}
\dot{x}(t) & =A x(t)+B(u(t)+f(t)) \\
y(t) & =C x(t),
\end{aligned}
$$


where $x(t) \in R^{n}$ is the system state, $u(t) \in R^{m}$ is the system control input, $y(t) \in R^{p}$ is the system output, $f(t) \in R^{m}$ is an unknown bounded exogenous disturbance, with $m \leq p<$ $n$. The system matrices $A, B, C$ are constant of appropriate dimensions.A sliding vector is to be constructed using only outputs as

$$
s=H y .
$$

Assumption 1: The disturbance $f(t)$ and its first and second derivatives are bounded.

Assumption 2: There exists a full rank $m \times p$ matrix $H$ such that the square matrix $H C B$ is invertible and the continuoustime sliding surface, $s(t)=0$, is legitimate [11], [12], i.e the reduced order motion is stable.

Remark 2.1: A necessary condition is system (1) has relative degree equal to one with stable invariant zeros and $B$ and $C$ have full rank [12]. A constructive framework for finding a choice of $H$ was reported in [12].

The sampled-data counterpart of (1) is

$$
\begin{aligned}
x[k+1] & =\Phi x[k]+\Gamma u[k]+d[k] \\
y[k] & =C x[k],
\end{aligned}
$$

where $\Phi=e^{A T}, \Gamma=\int_{0}^{T} e^{A \tau} d \tau B$, and the disturbance is $d[k]=$ $\int_{0}^{T} e^{A \tau} B f((k+1) T-\tau) d \tau$. Define $\bar{A}, \overline{\bar{A}}, \bar{B}, \overline{\bar{B}}$ as follows

$$
\begin{aligned}
\bar{A} & =\frac{1}{T}\left(\Phi-I_{n}\right) \\
\overline{\bar{A}} & =\frac{1}{T^{2}}\left(\Phi-I_{n}-T A\right), \\
\bar{B} & =\frac{\Gamma}{T}, \\
\overline{\bar{B}} & =\frac{1}{T}(\Gamma-T B),
\end{aligned}
$$

With the above definitions, the system matrices of the discretetime system (19) can be written as

$$
\begin{aligned}
\Phi & =I_{n}+T \bar{A}=I_{n}+T(A+T \overline{\bar{A}}) \\
\Gamma & =T \bar{B}=T(B+T \overline{\bar{B}})
\end{aligned}
$$

Note that

$$
\overline{\bar{A}}=\sum_{k=2}^{\infty} T^{k-2} \frac{A^{k}}{k !}=O(1) .
$$

Due to the sampling effect, the disturbance $d[k]$ in the sampleddata system contains unmatched components [2]. The following is a revised version of Lemma 1 in [3].

Lemma 2.1: If Assumption 2 holds, then

$$
\begin{aligned}
& d[k]=\Gamma f[k]+\frac{T}{2} \Gamma v[k]+T^{3} \Delta d[k] \\
& d[k]-d[k-1]=O\left(T^{2}\right), \\
& d[k]-2 d[k-1]+d[k-2]=O\left(T^{3}\right),
\end{aligned}
$$

where

$$
v(t)=d f(t) / d t
$$

and

$$
\begin{aligned}
\Delta d[k] & =\hat{M} v[k]+\frac{1}{T^{3}} \int_{0}^{T} e^{A \tau} B \int_{k T}^{(k+1) T-\tau} \int_{k T}^{\beta} \dot{v}(\sigma) d \sigma d \beta d \tau \\
& =O(1)
\end{aligned}
$$

Note that in 13

$$
\hat{M}=\left(-\frac{1}{12} A-\frac{T}{12} \overline{\bar{A}}\right) B=O(1) .
$$

The proof is presented in [4].

Our objective is to design an output feedback sliding mode control law that will offer a high accuracy of sliding mode and state regulation in the presence of the unknown disturbance.

\section{MAIN RESULTS}

Define a nonsingular transformation matrix as

$$
P_{1}=\left[\begin{array}{c}
M \\
H C
\end{array}\right],
$$

where $M$ is a full rank $(n-m) \times n$ matrix such that $M B=0$. Note the condition $M B=0$ implies $\operatorname{Range}\left(M^{T}\right)=\operatorname{Null}\left(B^{T}\right)$.

Remark 3.1: According to Assumption 2, $H C B$ is invertible. Thus, no rows of $H C$ lie in the null space of $B^{T}$. This implies the rows of $M$ are independent of those of $H C$. In other words, $P_{1}$ has full rank.

Partition the inverse of $P_{1}$ into submatrices $Q$ and $R$ as

$$
P_{1}^{-1}=\left[\begin{array}{ll}
Q & R
\end{array}\right]
$$

where $Q$ has $n-m$ columns.

The coordinate transformation $P_{1} x=[\xi, s]^{T}$ leads to the following "regular" form

$$
\left[\begin{array}{l}
\dot{\xi} \\
\dot{s}
\end{array}\right]=\left[\begin{array}{cc}
M A Q & M A R \\
H C A Q & H C A R
\end{array}\right]\left[\begin{array}{l}
\xi \\
s
\end{array}\right]+\left[\begin{array}{c}
0 \\
H C B
\end{array}\right](u+f) .
$$

Clearly, the sliding mode dynamics of system (17) are

$$
\dot{\xi}=M A Q \xi=A_{c} \xi
$$

in which matrix $A_{c}$ contains stable invariant zeros of [1], [12].

Now, consider the sampled-data formulation of the continuous-time system with matched disturbances in (1):

$$
\begin{aligned}
x[k+1] & =\Phi x[k]+\Gamma u[k]+d[k] \\
y[k] & =C x[k] \\
s[k] & =H y[k],
\end{aligned}
$$

where the disturbance $d[k]$ is defined in 13 in Lemma 2.1 and the output feedback sliding vector is prescribed in (2). In this section, we will study an output feedback sliding mode control law for the sampled-data system (19). The stability of the closed-loop system under the proposed control law will be discussed initially if the disturbance is not taken into account. Lastly, the accuracy of the sliding mode and the bounds on the state variables in the presence of external disturbances will be analyzed.

\section{A. Output Feedback Control Design}

Taking into account the coordinate transformation in 15 along with the relationship $\Phi=I+T \bar{A}, \Gamma=T \bar{B}$ yields

$$
\begin{aligned}
{\left[\begin{array}{c}
\xi[k+1] \\
s[k+1]
\end{array}\right]=} & {\left[\begin{array}{cc}
I_{n-m}+T M \bar{A} Q & T M \bar{A} R \\
T H C \bar{A} Q & I_{m}+T H C \bar{A} R
\end{array}\right]\left[\begin{array}{l}
\xi[k] \\
s[k]
\end{array}\right] } \\
& +\left[\begin{array}{c}
T M \bar{B} \\
T H C \bar{B}
\end{array}\right] u[k]+\left[\begin{array}{l}
d_{11}[k] \\
d_{12}[k]
\end{array}\right]
\end{aligned}
$$


where

$$
\left[\begin{array}{l}
d_{11}[k] \\
d_{12}[k]
\end{array}\right]=T\left[\begin{array}{c}
M \bar{B} \\
H C \bar{B}
\end{array}\right]\left(f[k]+\frac{T}{2} v[k]\right)+T^{3} P_{1} \Delta d[k] .
$$

Using the relationship $\bar{B}=B+T \overline{\bar{B}}$ and $M B=0$, we have

$$
\begin{aligned}
M \bar{B} & =O(T) \\
d_{11}[k] & =O\left(T^{2}\right) \\
d_{12}[k] & =O(T) .
\end{aligned}
$$

Rewrite the $s[k]$ dynamics in 20 as

$$
s[k+1]=\left(I_{m}+T \Omega_{2}\right) s[k]+T H C \bar{B} u[k]+g[k],
$$

where

$$
g[k]=T \Omega_{1} \xi[k]+d_{12}[k],
$$

and

$$
\begin{aligned}
& \Omega_{1}=H C \bar{A} Q \\
& \Omega_{2}=H C \bar{A} R .
\end{aligned}
$$

We see that $g[k]$ contains the state variables in $\xi[k]$ and the portion of disturbances lying in the control range space. By solving $s[k+1]=0$, we obtain the discrete-time equivalent control law [13]

$$
u^{e q}[k]=-\frac{1}{T}(H C \bar{B})^{-1}\left(\left(I_{m}+T \Omega_{2}\right) s[k]+g[k]\right),
$$

which is not physically realizable since it contains $g[k]$. On the other hand, $g[k]$ can be approximated by $g[k-1]$ which is calculated from 22

$$
g[k-1]=s[k]-\left(I_{m}+T \Omega_{2}\right) s[k-1]-T H C \bar{B} u[k-1] .
$$

The last statement in Lemma 2.1 shows that the disturbance $d[k]$ can be approximated by $2 d[k-1]-d[k-2]$. Based on this observation, we approximate $g[k]$ by $2 g[k-1]-g[k-2]$. Note that $g[k-1]$ and $g[k-2]$ can be computed by (27). Hence, replacing $g[k]$ by $2 g[k-1]-g[k-2]$ in (26, we obtain a new implementable control law

$$
\begin{aligned}
u[k]= & -\frac{1}{T}(H C \bar{B})^{-1}\left(\left(3 I_{m}+T \Omega_{2}\right) s[k]-\left(3 I_{m}+2 T \Omega_{2}\right) s[k-1]\right. \\
& \left.+\left(I_{m}+T \Omega_{2}\right) s[k-2]\right)+2 u[k-1]-u[k-2] .
\end{aligned}
$$

Remark 3.2: Note that the formula for $\Omega_{2}$ defined in 25 involves $\bar{A}$ from $(4)$ and $R$ from $(16)$. Hence, the control law in (28), depending on $\Omega_{2}$, is easily computed.

This control law contains quantities at three consecutive time instants and can be viewed as a dynamic compensator driven by $s[k]$.

Lemma 3.1: There exists a sampling period $T$ small enough such that

i $\operatorname{Rank}(\Gamma)=\operatorname{Rank}(B)$;

ii the matrix $H C \Gamma$ be invertible.

Proof: Define the $n \times n$ matrix $\Theta=\int_{0}^{T} e^{A \tau} d \tau$, then $\Gamma=\Theta B$. Let $J$ be the Jordan form of $A$ with eigenvalues $\lambda_{i}, i=1, \ldots, n$. Then,

$$
\operatorname{det}(\Theta)=\operatorname{det}\left(\int_{0}^{T} e^{A \tau} d \tau\right)=\operatorname{det}\left(\int_{0}^{T} e^{J \tau} d \tau\right)=\prod_{i=1}^{n} \int_{0}^{T} e^{\lambda_{i} \tau} d \tau .
$$

If all the eigenvalues of $A$ are real, then the determinant of $\Theta$ is nonzero, implying $\Theta$ nonsingular so that $\operatorname{Rank}(\Gamma)=$ $\operatorname{Rank}(\Theta B)=\operatorname{Rank}(B)$. Now consider a complex conjugate pair of eigenvalues $\lambda_{i}=\alpha+j \beta$ and $\lambda_{i}^{*}=\alpha-j \beta$ with $\beta \neq 0$. The determinant $\operatorname{det}(\Theta)$ has a factor

$$
\begin{array}{r}
\int_{0}^{T} e^{\lambda_{i} \tau} d \tau \int_{0}^{T} e^{\lambda_{i}^{*} \tau} d \tau=\frac{1}{\alpha^{2}+\beta^{2}}\left(e^{\lambda_{i} T}-1\right)\left(e^{\lambda_{i}^{*} T}-1\right) \\
=\frac{1}{\alpha^{2}+\beta^{2}}\left(\left(e^{\alpha T}-\cos \beta T\right)^{2}+\sin ^{2} \beta T\right),
\end{array}
$$

which is nonzero for any $T$ such that $|\beta T| \neq k \pi$, for a positive integer $k$. A possible choice of $T$ is to ensure $0<|\beta T|<\pi$. Hence, the matrix $\int_{0}^{T} e^{A \tau} d \tau$ is nonsingular, implying $\operatorname{Rank}(B)=\operatorname{Rank}(\Gamma)$. From (6),

$$
H C \Gamma=T H C \bar{B}=T H C(B+T \overline{\bar{B}})=T(H C B)+O\left(T^{2}\right) .
$$

Since $H C B$ is invertible, the matrix $H C \Gamma$ is invertible for $T$ sufficiently small.

\section{B. Stability Analysis}

The approach in III-A is different from [4] because two previous time instants are taken into account in the construction of the proposed control law (28). However, in this case, similar to [4], we study an augmented dynamic system which includes $\xi[k], s[k], s[k-1], u[k]$ and $u[k-1]$.

Introduce the following new variables:

$$
\begin{aligned}
s_{1}[k] & =s[k-1], \\
\gamma[k] & =\text { THCB } u[k], \\
\gamma_{1}[k] & =\text { THCB } u[k-1] .
\end{aligned}
$$

Thus, the dynamic control law 28 can be rewritten as

$$
\begin{aligned}
\gamma[k+1]= & -\left(3 I_{m}+T \Omega_{2}\right) s[k+1]+\left(3 I_{m}+2 T \Omega_{2}\right) s[k] \\
& -\left(I_{m}+T \Omega_{2}\right) s_{1}[k]+2 \gamma[k]-\gamma_{1}[k] .
\end{aligned}
$$

Substituting the variables $s[k+1]$ of $(22)$ and $g[k]$ of $(23)$ into (32) and assembling all the dynamic equations for the variables $\xi[k], s[k], s_{1}[k], \gamma[k]$, and $\gamma_{1}[k]$ yields the augmented system

$$
\left[\begin{array}{c}
\xi[k+1] \\
s[k+1] \\
s_{1}[k+1] \\
\gamma[k+1] \\
\gamma_{1}[k+1]
\end{array}\right]=A_{\text {aug }}\left[\begin{array}{c}
\xi[k] \\
s[k] \\
s_{1}[k] \\
\gamma[k] \\
\gamma_{1}[k]
\end{array}\right]+d_{2}[k],
$$

where

$$
A_{\text {aug }}=\left[\begin{array}{cc}
A_{s} & T N_{1} \\
T N_{2} & A_{f}
\end{array}\right]
$$

is the augmented system matrix with the sub-matrix

$$
A_{s}=I_{n-m}+T M \bar{A} Q=I_{n-m}+T A_{c}+T^{2} M \overline{\bar{A}} Q
$$

with $A_{c}$ given in (18), and

$$
A_{f}=\left[\begin{array}{cccc}
\left(I_{m}+T \Omega_{2}\right) & 0 & I_{m} & 0 \\
I_{m} & 0 & 0 & 0 \\
-\left(2 I_{m}+T \Omega_{2}\right) \Omega_{2} & -\left(I_{m}+T \Omega_{2}\right) & -\left(I_{m}+T \Omega_{2}\right) & -I_{m} \\
0 & 0 & I_{m} & 0
\end{array}\right] .
$$


The (augmented) disturbance term

$$
d_{2}[k]=\left[\begin{array}{c}
d_{11}[k] \\
d_{12}[k] \\
0 \\
-\left(3 I_{m}+T \Omega_{2}\right) d_{12}[k] \\
0
\end{array}\right]
$$

and the off diagonal matrices in 34 are

$$
\begin{gathered}
N_{1}=\left[\begin{array}{llll}
M \bar{A} R & 0_{(n-m) \times m} & M \overline{\bar{B}}(H C \bar{B})^{-1} & 0_{(n-m) \times m}
\end{array}\right], \\
N_{2}=\left[\begin{array}{llll}
\Omega_{1} & 0 & -\left(3 I_{m}+T \Omega_{2}\right) \Omega_{1} & 0
\end{array}\right]^{T} .
\end{gathered}
$$

Defining

$$
\bar{A}_{c} \triangleq A_{c}+T M \overline{\bar{A}} Q
$$

from (35), $A_{\text {aug }}$ can be written in the discrete-time singularly perturbed form as [14]

$$
A_{\text {aug }}=\left[\begin{array}{cc}
I_{n-m}+T \bar{A}_{c} & T N_{1} \\
T N_{2} & A_{f}
\end{array}\right] .
$$

Observe that $A_{f}^{4}=0$, implying the fast subsystem matrix $A_{f}$ is a nilpotent matrix of degree 4 . Employing the properties of nilpotent matrices, all the eigenvalues of $A_{f}$ are zero and $\operatorname{det}\left[I_{4 m}-A_{f}\right]=1$. To identify the magnitude of the eigenvalues of the singularly perturbed system matrix $A_{\text {aug }}$ in (39), introduce the following decoupling transformation to separate system (33) into distinct slow and fast subsystems [14]

$$
P_{2}=\left[\begin{array}{cc}
I_{n-m}-T J L & -T J \\
L & I_{4 m}
\end{array}\right], P_{2}^{-1}=\left[\begin{array}{cc}
I_{n-m} & T J \\
-L & I_{4 m}-T L J
\end{array}\right],
$$

in which the matrices $L \in R^{4 m \times(n-m)}$ and $J \in R^{(n-m) \times 4 m}$ are functions of the sampling period $T$ and satisfy

$$
\begin{aligned}
0= & T N_{2}+L(T)-A_{f} L(T)+T L(T)\left(\bar{A}_{c}-N_{1} L(T)\right), \\
0= & N_{1}+J(T)-J(T) A_{f}+T\left(\bar{A}_{c}-N_{1} L(T)\right) J(T) \\
& -T J(T) L(T) N_{1} .
\end{aligned}
$$

According to [14], $L$ and $J$ exist for sufficiently small values of $T$ since $I_{4 m}-A_{f}$ is nonsingular. In addition, application of the implicit function theorem shows that $L(T)$ and $J(T)$ are analytic at $T=0$. Setting $T=0$ in equations (41) yields

$$
\left(I_{4 m}-A_{f}\right) L(0)=0
$$

It follows that $L(0)=0$, and hence

$$
L=O(T) \text {. }
$$

Let

$$
\left[\begin{array}{l}
w[k] \\
\eta[k]
\end{array}\right]=P_{2}\left[\begin{array}{lllll}
\xi[k] & s[k] & s_{1}[k] & \gamma[k] & \left.\gamma_{1}[k]\right]^{T},
\end{array}\right.
$$

where $w[k] \in R^{n-m}$ and $\eta[k] \in R^{4 m}$. The coordinate transformation $P_{2}$ from (40) puts the augmented system dynamics into the fully decoupled two-time scale form

$$
\left[\begin{array}{l}
w[k+1] \\
\eta[k+1]
\end{array}\right]=\left[\begin{array}{cc}
\Phi_{s} & 0 \\
0 & \Phi_{f}
\end{array}\right]\left[\begin{array}{l}
w[k] \\
\eta[k]
\end{array}\right]+d_{3}[k]
$$

where

$$
\begin{aligned}
\Phi_{s} & =I_{n-m}+T \bar{A}_{c}-T N_{1} L \\
\Phi_{f} & =A_{f}+T L N_{1},
\end{aligned}
$$

and

$$
d_{3}[k]=P_{2}\left[\begin{array}{c}
d_{11}[k] \\
d_{12}[k] \\
0 \\
-\left(3 I_{m}+T \Omega_{2}\right) d_{12}[k] \\
0
\end{array}\right]=\left[\begin{array}{c}
O\left(T^{2}\right) \\
d_{12}[k]+O\left(T^{3}\right) \\
O\left(T^{3}\right) \\
-\left(3 I_{m}+T \Omega_{2}\right) d_{12}[k]+O\left(T^{3}\right) \\
O\left(T^{3}\right)
\end{array}\right] .
$$

We can now present one of the main results:

Theorem 3.1: Suppose Assumption 1 holds. In the absence of disturbances, the discrete-time output feedback sliding mode control law (28) renders the sampled-data system 20] asymptotically stable if the sampling period $T$ is small enough. Proof: The stability of the closed-loop system is decided by the eigenvalues of $\Phi_{s}$ and $\Phi_{f}$. From the definitions of $\bar{A}_{c}$ in (38) and $\Phi_{s}$ in (46), it can be seen that

$$
\lambda\left\{\Phi_{s}\right\}=1+T \lambda\left\{A_{c}+T M \overline{\bar{A}} Q-N_{1} L\right\} .
$$

Since $A_{c}$ contains stable eigenvalues of the zero dynamics of the original continuous-time sliding motion in (18) and $L=O(T)$, there exists a small $T$ such that the eigenvalues of $\left(A_{c}+T M \overline{\bar{A}} Q-N_{1} L\right)$ have negative real parts. Therefore, the eigenvalues of $\Phi_{s}$ lie in the unit circle for a sufficiently small $T$. On the other hand, let $q$ be an eigenvector corresponding to an eigenvalue of $\Phi_{f}$. We have

$$
\left(\lambda\left\{\Phi_{f}\right\}\right)^{4}\|q\|=\left\|\left(\lambda\left\{\Phi_{f}\right\}\right)^{4} q\right\|=\left\|\Phi_{f}^{4} q\right\| \leq\left\|\Phi_{f}^{4}\right\|\|q\| .
$$

This implies

$$
\left(\lambda\left\{\Phi_{f}\right\}\right)^{4} \leq\left\|\Phi_{f}^{4}\right\| .
$$

Since $A_{f}^{4}=0$ and $L=O(T)$, from 47 we have

$$
\Phi_{f}^{4}=\left(A_{f}+O\left(T^{2}\right)\right)^{4}=O\left(T^{2}\right) .
$$

Hence, 51 implies that

$$
\lambda\left\{\Phi_{f}\right\}=O(\sqrt{T}) .
$$

This shows that the eigenvalues of $\Phi_{f}$ lie in the unit circle for a sufficiently small $T$.

Remark 3.3: The proof above uses an argument that if a matrix $S$ is stable in the sense of continuous-time systems, then $\bar{S}=I+T S$ is stable in the sense of discrete-time systems provided $T$ is small enough. Indeed, let $\lambda\{S\}=-\alpha+j \beta$ where $\alpha>0$. Then,

$$
\lambda\{\bar{S}\}=1-T \alpha+j T \beta .
$$

If $T$ is chosen such that

$$
T \leq \frac{2 \alpha}{\alpha^{2}+\beta^{2}}
$$

then $|\lambda\{\bar{S}\}| \leq 1$. Hence, there exists a sufficiently small $T$ such that the eigenvalues of $\Phi_{s}$ lie in the unit circle.

\section{Accuracy Analysis}

In this subsection, we will investigate the accuracy issue of the sliding motion and the bound of the state vector when the system is subject to the external disturbance.

Theorem 3.2: Under Assumptions 1 and 2, the sampled-data output feedback control (28) produces a quasi-sliding motion on the sliding surface $s(t)$ with an $O\left(T^{3}\right)$ boundary layer. 
Furthermore, the ultimate bound of the original state variables and the control input are $O\left(T^{2}\right)$ and $O(1)$ respectively.

Proof: From (33) and (34), we have

$$
\left[\begin{array}{c}
\xi[k+4] \\
s[k+4] \\
s_{1}[k+4] \\
\gamma[k+4] \\
\gamma_{1}[k+4]
\end{array}\right]=A_{\text {aug }}^{4}\left[\begin{array}{c}
\xi[k] \\
s[k] \\
s_{1}[k] \\
\gamma[k] \\
\gamma_{1}[k]
\end{array}\right]+d_{4}[k]
$$

where

$$
\begin{aligned}
& d_{4}[k]=\left[\begin{array}{lllll}
d_{41}[k] & d_{42}[k] & d_{43}[k] & d_{44}[k] & d_{45}[k]
\end{array}\right]^{T} \\
& =d_{2}[k+3]+A_{\text {aug }} d_{2}[k+2]+A_{\text {aug }}^{2} d_{2}[k+1]+A_{\text {aug }}^{3} d_{2}[k],
\end{aligned}
$$

where $d_{2}[k]$ is given in 37 . Hence, after some computations,

$$
\begin{aligned}
d_{41}[k]= & d_{11}[k+3]-2 d_{11}[k+2]+d_{11}[k+1] \\
& +T M \bar{A} R\left(d_{12}[k+2]-d_{12}[k+1]\right)+O\left(T^{3}\right), \\
d_{42}[k]= & d_{12}[k+3]-2 d_{12}[k+2]+d_{12}[k+1]+O\left(T^{3}\right), \\
d_{43}[k]= & d_{12}[k+2]-2 d_{12}[k+1]+d_{12}[k]+O\left(T^{3}\right), \\
d_{44}[k]= & -\left(3 I_{m}+T \Omega_{2}\right) d_{12}[k+3]+\left(3 I_{m}+2 T \Omega_{2}\right) d_{12}[k+2] \\
& -\left(I_{m}+T \Omega_{2}\right) d_{12}[k+1]+O\left(T^{3}\right),
\end{aligned}
$$

and

$$
\begin{aligned}
d_{45}[k]= & -\left(3 I_{m}+T \Omega_{2}\right) d_{12}[k+2]+\left(3 I_{m}+2 T \Omega_{2}\right) d_{12}[k+1] \\
& -\left(I_{m}+T \Omega_{2}\right) d_{12}[k]+O\left(T^{3}\right) .
\end{aligned}
$$

Applying the transformation (40) to 55 and using Lemma 2.1. with $d_{11}[k]$ and $d_{12}[k]$ playing the role of $d[k]$, we have

$$
\left[\begin{array}{c}
w[k+4] \\
\eta[k+4]
\end{array}\right]=\left[\begin{array}{cc}
\Phi_{s}^{4} & 0 \\
0 & \Phi_{f}^{4}
\end{array}\right]\left[\begin{array}{c}
w[k] \\
\eta(k)
\end{array}\right]+\left[\begin{array}{c}
O\left(T^{3}\right) \\
O\left(T^{3}\right) \\
O\left(T^{3}\right) \\
O(T) \\
O(T)
\end{array}\right]
$$

At steady state, we obtain $w[k+4] \approx w[k]$. Hence,

$$
\left(I_{n-m}-\Phi_{s}^{4}\right) w[k]=O\left(T^{3}\right) .
$$

Since $I_{n-m}-\Phi_{s}^{4}=-4 T A_{c}+O\left(T^{2}\right)$ and $\lambda\left\{A_{c}\right\} \neq 0$, for small enough $T$, we attain

$$
w[k]=O\left(T^{2}\right)
$$

Similarly, $\eta[k+4] \approx \eta[k]$ at steady state. From (36) and (57), we have

$$
\left(I_{4 m}-O\left(T^{2}\right)\right) \eta[k]=\left[\begin{array}{c}
O\left(T^{3}\right) \\
O\left(T^{3}\right) \\
O(T) \\
O(T)
\end{array}\right], \text { or } \eta[k]=\left[\begin{array}{c}
O\left(T^{3}\right) \\
O\left(T^{3}\right) \\
O(T) \\
O(T)
\end{array}\right] .
$$

Using (58) and 59, we obtain

$$
\left[\begin{array}{c}
\xi[k] \\
s[k] \\
s_{1}[k] \\
\gamma[k] \\
\gamma_{1}[k]
\end{array}\right]=P_{2}^{-1}\left[\begin{array}{l}
w[k] \\
\eta[k]
\end{array}\right]=\left[\begin{array}{c}
O\left(T^{2}\right) \\
O\left(T^{3}\right) \\
O\left(T^{3}\right) \\
O(T) \\
O(T)
\end{array}\right] .
$$

This shows that the accuracy of the sliding mode obtained is of order of $O\left(T^{3}\right)$, i.e. $s[k]=O\left(T^{3}\right)$. The ultimate order of the original state variables is given by

$$
x[k]=P_{1}^{-1}\left[\begin{array}{ccccc}
I_{n-m} & 0 & 0 & 0 & 0 \\
0 & I_{m} & 0 & 0 & 0
\end{array}\right] P_{2}^{-1}\left[\begin{array}{c}
w[k] \\
\eta[k]
\end{array}\right]=O\left(T^{2}\right),
$$

and 60 shows that the ultimate bound of $\gamma[k]$ is $O(T)$. This implies the ultimate bound of $u[k]$ is $O(1)$.

Remark 3.4: In [7], [4], $O\left(T^{2}\right)$ accuracy is achieved for the boundary layer in the sliding mode, and $O(T)$ bounds for the state vector. The approach in this paper provides better bounds: $O\left(T^{3}\right)$ and $O\left(T^{2}\right)$ respectively.

\section{NUMERICAL EXAMPLE}

Here we use the lateral dynamics of an aircraft from [15] to illustrate the proposed approach. The system matrices are

$$
A=\left[\begin{array}{cccc}
-3.79 & 0.04 & -52 & 0 \\
-0.14 & -0.36 & 4.24 & 0 \\
0.06 & -1 & -0.27 & 0.05 \\
1 & 0.06 & 0 & 0
\end{array}\right], B=\left[\begin{array}{ccc}
25 & 9.83 \\
1.42 & -4.2 \\
0.01 & 0.05 \\
0 & 0
\end{array}\right], C=\left[\begin{array}{llll}
1 & 0 & 0 & 0 \\
0 & 1 & 0 & 0 \\
0 & 0 & 0 & 1
\end{array}\right]
$$

The state vector consists of roll rate, yaw rate, sideslip, and bank angle. The control vector represents the aileron and rudder deflections. Roll rate, yaw rate, and bank angle are taken as the output vector.

The invariant zero of the system is -0.1796 . The remaining eigenvalue for the sliding mode is chosen as -2 . Using the method in [12], we obtain

$$
H=\left[\begin{array}{ccc}
0.035306 & 0.082634 & 0.076550 \\
0.011937 & -0.210157 & 0.008324
\end{array}\right] \text {. }
$$

In all the simulations, the initial condition is given by $x(0)=[-1,2,1,-2]^{T}$. The sampling period is $T=0.1 s$, and the disturbance vector $f(t)=\left[\begin{array}{ll}1+\sin (0.5 t) & 0.5 \cos (t)\end{array}\right]^{T}$ only affects the system dynamics from $t=10 \mathrm{~s}$ onwards.

For comparison, the method presented in [4] was also implemented. Figures 1, 3, 5 demonstrate the method in [4], while the remaining figures illustrate the method proposed in this paper. The $O(T)$ state regulation accuracy and the $O\left(T^{2}\right)$ quasi-sliding motion are shown in Figures 1 and 3 . The proposed control law (28) leads to an ultimate state regulation error magnitude of $O\left(T^{2}\right)$ as shown in Fig. 2 Fig. 4 illustrates the associated quasi sliding motion whose boundary layer has a numerical value of $O\left(T^{3}\right)$, which agrees with the theoretical bound in Theorem 3.2. The control efforts for the method in [4] and the method proposed in the paper are plotted in Fig. 5 and Fig. 6, and show similar bounds.

In this example, the ultimate bounds on the state variables and the accuracy of the sliding motion for the new method proposed in this paper are better than those for the method presented in [4]. This agrees with the earlier analysis.

\section{CONCLUSION}

A new method has been presented to solve the problem of output feedback sliding mode control for sampled-data systems. The proposed scheme is able to achieve better accuracy than earlier work: a quasi sliding motion with an $O\left(T^{3}\right)$ boundary layer, and an $O\left(T^{2}\right)$ ultimate bound for state 


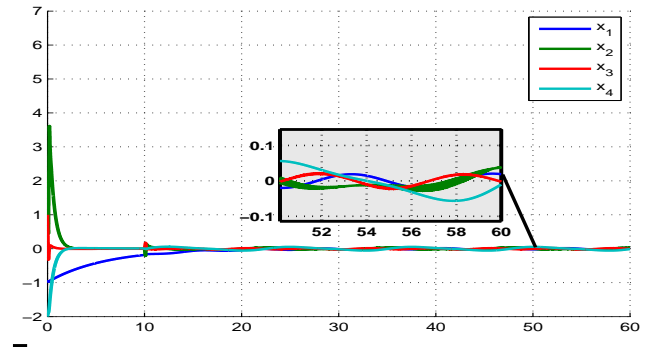

Fig. 1. The evolution of the state variables for the method in [4]

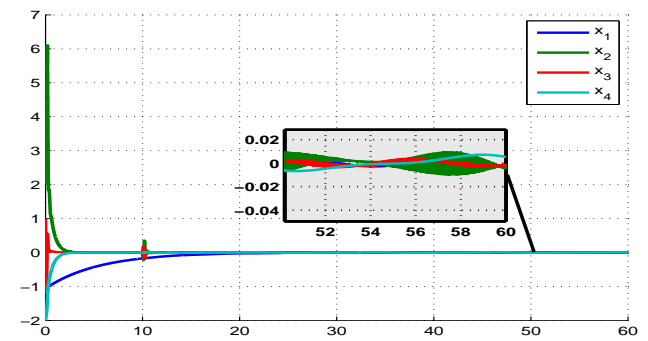

Fig. 2. The evolution of the state variables for the proposed method

regulation. The effectiveness of the new method was illustrated by a numerical example.

In practice, the model of a system may neglect the presence of unmodeled inertial dynamics, which may be the consequence of neglecting the dynamics of the transducers. In this case, the characteristics of the proposed scheme may be affected. This is a possible future research topic.

\section{REFERENCES}

[1] C. Milosavljevic, General conditions for the existence of quasi-sliding mode on the switching hyper-plane in discrete variable structure systems, Automat. Remote Contr. 46 (3) (1985) 307-314.

[2] W.-C. Su, S. V. Drakunov, Ü. Özguner, An $O\left(T^{2}\right)$ boundary layer in sliding mode for sampled-data systems, IEEE Trans. Autom. Control 45 (2000) 482-485.

[3] K. Abidi, J.-X. Xu, Y. Xinghuo, On the discrete-time integral slidingmode control, IEEE Trans. Autom. Control 52 (2007) 709-715.

[4] T. Nguyen, W.-C. Su, Z. Gajic, Output feedback sliding mode control for sampled data systems, IEEE Trans. Autom. Control 55 (2010) 16841689.

[5] N. O. Lai, C. Edwards, S. K. Spurgeon, On output tracking using dynamic output feedback discrete-time sliding-mode controllers, IEEE Trans. Autom. Control 52 (2007) 1975-1981.

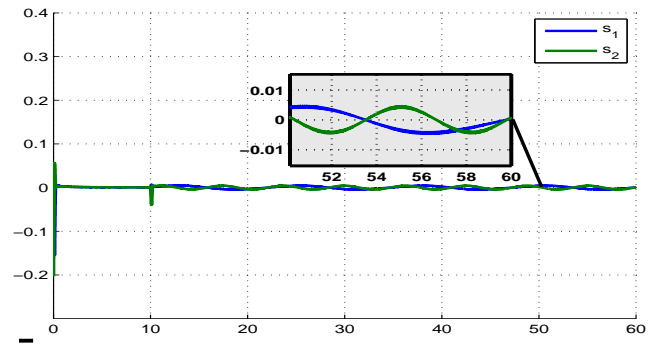

Fig. 3. The evolution of the sliding motion for the method in [4]

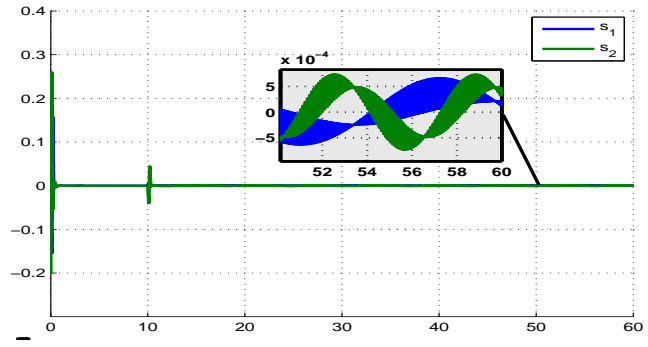

Fig. 4. The evolution of the sliding motion for the proposed method

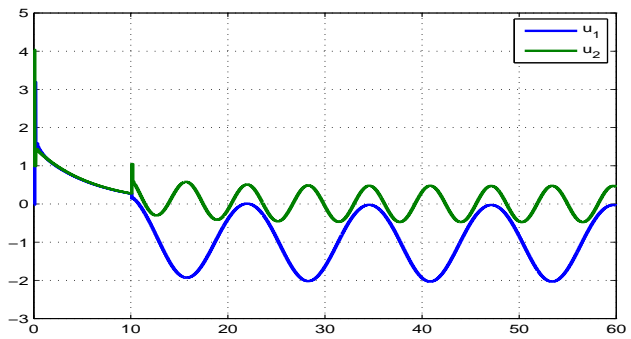

Fig. 5. The evolution of the control effort for the method in [4]

[6] C. Milosavljevic, B. Perunicic-Drazenovic, B. Veselic, Discrete-time velocity servo system design using sliding mode control approach with disturbance compensation, IEEE Trans. Ind. Informat. 9 (2013) 920-927.

[7] J.-X. Xu, K. Abidi, Discrete-time output integral sliding-mode control for a piezomotor-driven linear motion stage, IEEE Trans. Autom. Control 55 (2008) 3917-3926.

[8] D. Mitic, C. Milosavljevic, Sliding mode-based minimum variance and generalized minimum variance controls with $O\left(T^{2}\right)$ and $O\left(T^{3}\right)$ accuracy, Electrical Engineering 86 (2004) 229-237.

[9] T. Nguyen, W.-C. Su, Z. Gajic, On $O\left(T^{2}\right)$ state regulation with output feedback sliding mode control for sampled-data systems, in: Proc. of the American Control Conference, Baltimore, USA, 2010, pp. 1823-1828.

[10] P. Kokotovic, H. Khalil, J. O'Reilly, Singular Perturbation Methods in Control: Analysis and Design, Academic Press, Orlando, FL, 1986.

[11] R. EL-Khazali, R. DeCarlo, Output feedback variable structure control design, Automatica 31 (1995) 805-816.

[12] C. Edwards, S. Spurgeon, Sliding mode stabilization of uncertain systems using only output information, Int. J. Cont. 62 (1995) 1129-1144.

[13] V. I. Utkin, S. Drakunov, On discrete-time sliding mode control, in: Pro IFAC Symp. Nonlinear Contr. Syst., Capri, Italy, 1989, pp. 484-489.

[14] B. Litkouhi, H. Khalil, Multirate and composite control of two-timescale discrete-time systems, IEEE Trans. Autom. Control 30 (1985) 645651.

[15] S. Srinathkumar, Eigenvalue/eigenvector assignment using output feedback, Tech. Rep. 1118, Langley Research Center, Hampton, Virginia (Feb 1978).

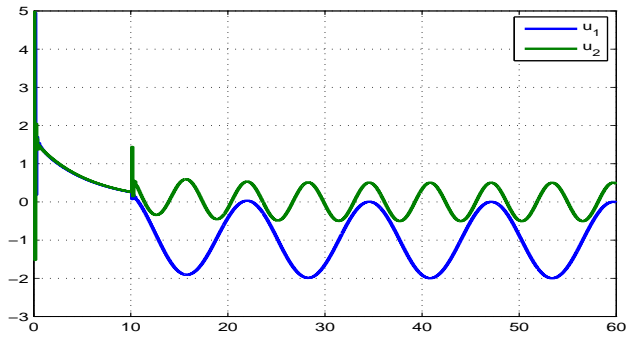

Fig. 6. The evolution of the control effort for the proposed method 